W. KUSSY / ELEKTRISCHE ANTRIEBE 

DR. ING. WERNER KUSSY

\title{
ELEKTRISCHE ANTRIEBE
}

\author{
VON HEBEZEUGEN
}

\section{UND TRANSPORTANLAGEN}

Mit 186 Tevabbildungen

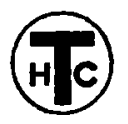

TECHNISCHER VERLAG HERBERT CRAM , BERLIN W 35

1954 
Satz: Walterde Gruyter \& Co., Berlin W 35

Gedrucktbei Ottovon Holten in Berlin 